\title{
Evaluating and Retrieving Parameters for Optimizing Organizational Structures in Real Estate and Construction Management
}

\author{
Wolfgang Eber ${ }^{1 *}$, Josef Zimmermann ${ }^{1}$ \\ 1 Department of Civil, Geo and Environmental Engineering, TUM - Technical University of Munich, Arcisstrasse 21., \\ 80333 Munich, Germany \\ * Corresponding author, e-mail: W.Eber@bv.tu-muenchen.de
}

Received: 18 June 2018, Accepted: 27 August 2018, Published online: 06 November 2018

\begin{abstract}
All organization is about creating structures. In particular, unique projects as are typical for Real Estate and Construction Management issues require a custom tailored organization due to the nonrecurring character. Moreover, as they are generally of large volume and traditionally tightly framed only an exactly fitting organization is capable to deal with the naturally given set of unknown parameters. Therefore, the term of Risk Management describes nothing more than an efficient organization where the treatment of varying parameters, i.e. risks, is well defined and prepared on an abstract level. Further abstraction of the question of an appropriate structure leads to principle considerations regarding the qualities of a structure, based on the fundament of Theory of Systems. Classical optimization methods are bound to fail here since they typically rest on the assumption of a given structure and are not flexible enough to create improved versions. However, parameterizing structures by the values of complexity, heterogeneity and recursiveness provide well-established assessment of the sensibility of organization structures regarding their stability, even against externally driven variations of internal variables. Thus, such parameters need to be retrieved from a given system and analyzed in order to allow judging the system's behavior in the long run as well as can be used for constructing add-ons to the structures in order to improve the efficiency of the organization. This focusses on encompassing characteristics like reducing complexity, increasing separability and stabilizing by the introduction of controlling elements. This paper is the revised version of the paper that has been published in the proceedings of the Creative Construction Conference 2018 (Zimmermann and Eber, 2018).
\end{abstract}

\section{Keywords}

complexity, construction projects, hierarchical structures, optimization, real estate projects

\section{Principles of Optimizing Organizational Structures Section}

Recent projects in Real Estate and Construction Management are becoming larger, therewith taking in more interacting participants, consuming more and more different resources, and are to be realized within shorter timeframes than ever before (Lewis, 2002). After long initial phases of design, planning, negotiation of permissions and optimizing procedures they are to be conducted flawlessly in shortest time and to produce no surprising events due to risky issues and in particular no repetitive loops (Straub, 2014; Zimmermann et al., 2014). Thus, part of the preparation phase is to establish meticulously optimal structures, processes and parameters in order to ensure proper operation of the construction or development phase (Schelle et al., 2005; Schulte-Zurhausen, 2002). Since careful preparation is under all circumstances much less costly than later reconsideration, special attention needs to be put on optimal design of the operation to come. In this paper, optimization parameters for the organizational structures are to be derived and proposed for ad hoc use.

As an extension to the paper Zimmermann and Eber (2018), details of retrieving and measuring such parameters from real projects are discussed here. This, inserted into Section 2.2 for each parameter, allows for explicit use and judgement of the possible performance and efficiency as well as for constructing additional structures improving given organizational situations. 


\subsection{General Remarks}

Optimization methods are generally based on manually decomposing the complexity of a problem into a sensible structure. This is to be further broken down into finer structures where finally an element is composed by a single variable (Gordon and Helmer, 1964). An accordingly well-formulated system can be described by the state-vector and the transfer-function of how a state affects the consecutive state (von Bertalanffy, 1969; Luhmann, 1984).

If one variable is declared a preference-value, respective algorithms are available to modify the system-state until the preference-value is optimized, i.e. maximized or minimized. So far, this can be achieved even under further boundary conditions at least by numerical approaches.

In particular, static systems may be optimized where no temporal development needs to be taken into account; namely, the character of approaching optimal system-states plays no role. Dynamic systems may include time as an additional one-way developing variable. As long as the development can be determined by additional system-variables like the speed of modification or the strength of an assignment, e.g. as a definition of the character of controlling in force and latency, also dynamic aspects can be modelled and optimized.

Yet, this poses the problem that the structure of the system itself is not subject of the optimization consideration. However, exactly structures, e.g. regarding responsibilities, delegation, reporting and instructing as well as control loops, mainly define the stability of a system developing on the time axis. Thus, the question arises of how structures may be optimized, possibly prior to parameter optimization.

Recent approaches work with sensible predefined organization types like trees and focus on finding minimal structures based on the goal to minimize the number of interfaces as these are expected to induce loss of information. Others propose principally specific structures as they avoid loops and therefore give no room to exponential or oscillating behavior. Some very classical approaches address the problem of optimal organizational structures by modelling all possible structures as complete graphs and optimize the degree of assignment as parameters on the structure. Examples would be the transportation problem or, as a derivative, the 1-0 assignment. It is common to them, that possible assignments need to be given manually and associated with cost. The algorithm may then make use of total or partial assignments due to an overall given optimization-parameter, e.g., under the precondition of finding a tree-structure, the optimal tree spanning a set of nodes can be found. Such is achieved by either randomly or systematically modifying the structure throughout the available space of states. Finally, evaluating for the best option reveals the preferred scenario.

\subsection{Exemplary Structural Restrictions}

The classical algorithm of Ford (Kerzner, 2003; Schelle et al., 2005) sorts given activities according to their rank and allows for no degrees of structural freedom. Neither loops nor ambiguities are permitted. Thus, two problems need to be solved: On the one hand, rank-loops are existing and lead to some well measurable fuzziness with respect to time. This may not impede the algorithms but should result in clearly given values. On the other hand, a multiple set of scenarios may be given and needs to be evaluated for optimal structures based on ambiguous relationships. Such are given e.g. by relationships forcing activities to be executed anytime, but not concurrently. By now, they are modelled introducing an arbitrary sequence of one activity preceding the other, but based on no reason. So half of the scenarios are not investigated and need to be tackled by manual override.

The term "Optimization of structures" is widely understood as optimizing physical structures (Straub, 2014) where every volume elements is strongly impacted by the surrounding elements in contrast to organizational structures. One of the most promising approaches is based on bionic evolutionary methods. While iteratively checking for the distribution of strain and stress, some parts of the structure are growing, some are diminishing accordingly until an even distribution of loads and bearing forces is achieved. This approach corresponds to classical algorithms, e.g. derived from the transport or the assignment algorithms where all encompassing structures of maximum complexity are being subjected to optimization by rules of reducing the parameterized strength of an interaction until the criterion of optimization is met.

\subsection{Requirements}

On this background, the principle requirements to develop sensible organizational structures can be formulated. First of all, the structure needs to mirror reality and thus must not be restricted by algorithmic imperfections. This needs primarily to be observed when analyzing existing structures, e.g. company teams or markets. Yet, if systems ready to accomplish a task need to be constructed as in a project team, some restrictions of the structures are not so much introduced by the abilities of an algorithm but by the problem to be solved itself. In this case, criteria like complexity and 
stability come into play (White et al., 2004; Zimmermann and Eber, 2012; 2014).

\section{Principle View on Optimizing Structures}

Optimal structures themselves are only in some very rare cases subject to the particular application, e.g. for legal issues. Mostly advantages or disadvantages of a structure are determined by the possible outcome of the behavior of the given system.

\subsection{Behavior of a System}

Let a general system be given as a set of interacting elements (von Bertalanffy, 1969; Wasserman and Faust, 1994; Zimmermann and Eber, 2017):

$\Omega=\left\{n_{i}, k_{j} \mid i=1 . . N, j=1 . . K, K \simeq N^{1+\alpha}\right\}$.

Every element $n_{i}$ may employ interdependencies to every other element (Gordon and Helmer, 1964) causing complex behavior, which is described by a set of differential equations

$n_{i}: \frac{\partial Q_{i}}{\partial t}=f_{i}\left(Q_{r}\right) \mid r=1 . . N \quad k_{j}:\left\{\left(n_{i} \rightarrow n_{r}\right) \mid i, r=1 . . N\right\}$.

These are generally solved by complex exponential systems of the form (Haken, 1983; White et al., 2004; Wiener, 1992):

$Q_{i}=\sum_{j} g_{i, j} e^{\lambda_{j} t}\left(+\sum_{j} g_{i, j}^{\prime} e^{2 \lambda_{j} t}+\ldots\right)$

As a complex system is described by a set of linear differential equations where the solutions are complex exponential functions, the behavior is dominated by exponential escalation or oscillation. The share of exponentially decreasing variables is naturally low because the therefore required simplicity of a node referring mainly to itself with a negative coupling factor is rarely found. Nevertheless, such form the dissipative factors, which are in general responsible for the stability of a system (Zimmermann and Eber, 2017). Thus, depending on the sign and value of the coupling parameters $\lambda$, solutions reflect a set of more or less strongly coupled oscillators where the behavior is known to be of chaotic character. Since the entirety of interdependencies, i.e. the "complexity", represents the coupling parameters of the single differential equations, clearly the unpredictability of the system develops with complexity.

On this background, the terms and parameters of complexity need to be investigated in order to reflect on the sensibility of a given or constructed structure, in particular with regard to the sensitivity against modifications and time related development.

\subsection{Parameters of Complexity}

\subsubsection{Heterogeneity}

Homogeneous systems are represented by valid statistical momenta for e.g. in-degree or out-degree of nodes. Distributions are in particular given as e.g. Gaussian or Poissonian curves. In contrast, distributions with a heavy tail may be described by power laws $P(k)=a k^{-\gamma}$ (Caldarelli and Vespignani, 2007). Clearly, they cannot be represented by average values like the mean value or the variance if the exponent is small enough. Thus, the indicator of homogeneity, rsp. heterogeneity, is the exponent $\gamma$ :

- Heterogeneous: $\gamma<2\left(\nexists \bar{k}, \sigma^{2}\right)$

- Inbetween: $2<\gamma<3\left(\exists \bar{k}, \nexists \sigma^{2}\right)$

- Homogeneous $3<\gamma\left(\exists \bar{k}, \sigma^{2}\right)$.

In order to determine the strong heterogeneity limit, the mean value of the degree distribution is to be calculated $\bar{k}=\int_{1}^{\infty} k P(k) d k=\frac{a}{2-\gamma}\left(\infty^{2-\gamma}-1\right)$.

If $\gamma>2$ the exponent becomes negative, leading to the first term to approach a very small value to a positive power, which is zero, while the second term remains one.

Thus, with $\gamma>2$, the system is well represented by the mean value and thus called homogeneous. Otherwise, a system where $\gamma<2$ would be characterized by a heavy tail indicated by $\bar{k}=\infty$ and be called heterogeneous. Establishing a weaker limit focusses on the determination of the second momentum (variance) which is $\sigma^{2}=\int_{0}^{\infty}(k-\bar{k})^{2} P(k) d k$.

However, the term with the highest exponent under the integral will be of the type $k^{2} P(k) d k$ leading to the same consideration with a given limit of $\gamma=3$. In both cases, this does not imply that such values $k, \sigma^{2}$ are not existing, only, that they are not representing the given structure.

Remark: A large number of surveyed real systems are in fact exhibiting values close to the limit $2<\gamma<3$ (Barabási and Albert, 1999; Newman, 2003; Strogatz, 2001).

Determination of the heterogeneity-coefficient $\gamma$ for a given system can easily be achieved by calculating the linear regression of a double logarithmic gradient.

Remark: This approach applies to scale-free networks, as organizational systems would in general be. If not, as only the fat tail on the right side of the distribution is of particular interest, only the terms $k \geq \bar{k}$ are to be taken into account. 
$\gamma=-\frac{\sum_{k}(\ln k-\overline{\ln k})(\ln H(k)-\overline{\ln H(k)})}{\sum_{k}(\ln k-\overline{\ln k})^{2}}$

where $H(k)$ is the frequency of occurrence of the degree $k$ (in absolute numbers or possibly weighted). Due to the logarithmic handling of the values special care needs to be taken for terms where $k=0$ or $H(k)=0$.

The term $k=0$ may well be occurring for a zero degree but leads to a numerator of negative infinity. As these nodes are not participating the interdependencies, they may be ignored. Furthermore, these are in most cases located left of $\bar{k}$ and therefore of no relevance. Based on the same reason nodes, which are not interacting, i.e. $H(k)=0$ may be as well ignored. Yet, the fact needs to be kept in mind that buffering elements are thus neglected and only the share of interacting elements are considered. This is sensible as the possible heterogeneity given by some (few) nodes of higher degree is more important to be detected than a set of degrees, which are not at all connected. Thus, heterogeneity is systematically overestimated for good reasons. As an alternative method to be considered, the frequency distribution may probably be smoothed due to the discrete character of the given classes. This process is equivalent to the utilization of slightly larger classes and therefore clearly applicable. Yet, since the focus lies on the impact of highly connected nodes and not on the averages, the first approach is to be preferred.

\subsubsection{Complexity}

The term of complexity is widely understood only semantically, yet not defined mathematically. In particular needs to be distinguished whether a system is complex or merely

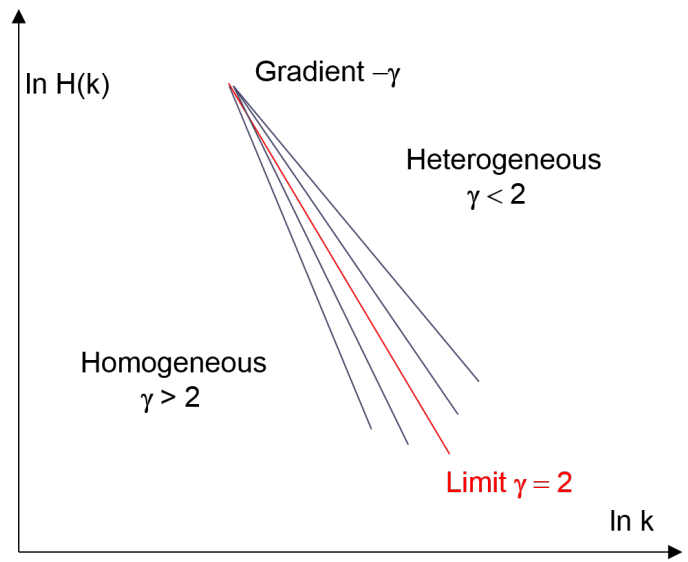

Fig. 1 Heterogeneity $\gamma$ derived from a double logarithmic plot complicated. According to (Caldarelli and Vespignani, 2007) at least two criteria need to be met to establish complexity. A complex system shows heterogeneity over all scales and emergent behavior. Furthermore, as emergent behavior is limited by the characteristic of being not reducible (Luhmann, 1984), complexity might be understood as property of a system which vanishes to some degree if reduced. Thus, complicated systems can be understood by reducing those to smaller (minimal) subsystems. Possible definitions of complexity, which are completely compatible with each other, are given here.

Complexity may be understood as the dimension of the configuration space of a project structure (Zimmermann and Eber, 2010; 2012). Let the elements of a system fill the system volume and order these in a way that each interaction to another element is understood as a next neighbor interface. The dimension of the volume scaled to a maximum dimensionality of 1 can be written as $\alpha=\ln (\xi+1) / \ln N=\ln (K / N+1) / \ln N$, where $N$ is the number of elements and $K$ the number of interactions, possibly normalized and weighted.

Similarly, complexity represents the average entropy of a node in comparison to the possible entropy according to Shannon (1948). The average number of choices for a node to influence is $(v+1)$ (av. edges incl. self), i.e. the number of real adjacent nodes, while the maximum number of choices, rsp. of adjacent nodes, is $N$ (each node incl. self). Then the information content per node is: $E=\ln (v+1)$ while the relative information content per node is $E_{R}=\ln (v+1) / \ln N=\alpha$. Finally, the entropy $S$ as the expectation value is also:

$S=-\sum p_{i} \ln p_{i}=\ln (v+1)$.

Alternatively, the complexity $\alpha$ is given as the exponent of the structural development of a modification $T$ from one layer $r$ to the next $r+1$. Thus, it reflects the degree of the linearity of the structural development $T(r) \simeq \xi^{1+\alpha r} \Delta(\zeta) /(1-\beta)$ with increasing structural steps $r$ and the positive factor with each step $\omega \simeq \xi^{\alpha}$ (Zimmermann and Eber, 2014; Zimmermann et al., 2014).

This can be made use of to retrieve the real complexity of a system, even if $\beta \neq 0$ since this term cancels considering a singular development step. So introducing a minor modification $\delta_{0}$ to a variable, the observed variance after $k$ logical steps $\delta_{k}$ returns $\omega^{k}=\left(\delta_{k} / \delta_{0}\right)$ and thus $\xi^{\alpha}=\omega=\left(\delta_{k} / \delta_{0}\right)^{1 / k}$ and therewith

$\alpha=\frac{\ln \left(\delta_{k} / \delta_{0}\right)}{k \ln \xi}$. 


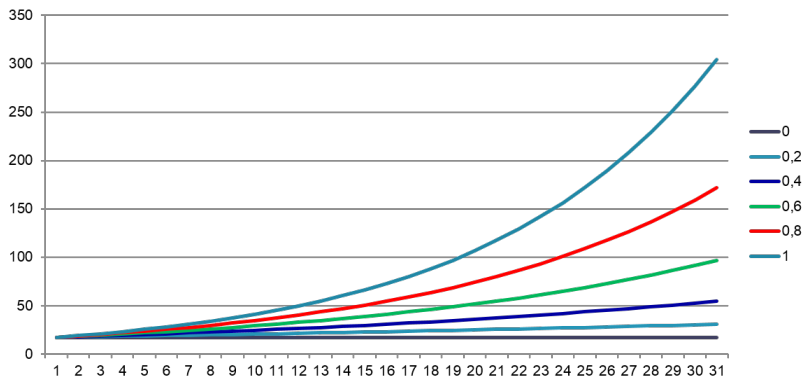

Fig. 2 Potential development of a deviation along causal ranks with a factor $\omega \simeq \xi^{\alpha}$ per step.

Over all, the understanding of "Complexity" comprises both the value of $\alpha$ representing the average structural interdependency and the heterogeneity $\gamma$ as an indicator of to which degree $\alpha$ is equally spread all over the system or concentrated to specific locations.

\subsubsection{Recursiveness}

Within iterative systems, complexity is not only given by the number of interactions $v s$. the available number of interactions but also by the repetitiveness of interactions to be utilized. Such is determined by the parameter of recursiveness, given by the number of (possibly weighted) paths leading from an element back to itself:

$$
(1 / N) \operatorname{Tr} \sum_{m=1}^{\infty} A_{i, i}^{m}=\beta
$$

where $N$ is the number of elements and $A_{i, j}$ the normalized weighted adjacency matrix. The value $\beta$ then represents the averaged percentage of an influence returning to the very same node. Thus, according to the understanding of complexity as the exponent of the development from step to step, repeated steps with a factor of $\beta$ to the power of the index of the iteration needs to be considered:

$$
\begin{aligned}
Z_{i+1} & =\xi^{\alpha} Z_{i} \Rightarrow Z_{i+1}=\xi^{\alpha} Z_{i} \beta^{0}+\xi^{\alpha} Z_{i} \beta^{1}+\xi^{\alpha} Z_{i} \beta^{2}+\ldots \\
& =Z_{i} \xi^{\alpha} /(1-\beta) .
\end{aligned}
$$

On this background, the basic complexity $\alpha$ needs to be modified to include the effects of the recursiveness $\beta$ :

$\xi^{\alpha^{(R)}}=\xi^{\alpha} /(1-\beta) \Rightarrow \alpha^{(R)}=\alpha-\ln (1-\beta) / \ln \xi$.

Zero recursiveness leads therefore to no effect while higher recursiveness $\beta \leq 1$ leads to significant increase of complexity. In particular needs to be noted that the complexity possibly rises to values greater than unity since $\alpha=1$ indicates the utilization of all possible interactions just once and not repeatedly.

Remark: Overall recursiveness obviously increases complexity as it possibly leads to unpredictable behavior.
This is according to the higher degree of the differential equation system allowing for chaotic oscillation and escalating values. Therefore, the reaction of a system on modifications and the immediate as well as the long-term stability are mainly determined by recursiveness. Since in this context no general rules concerning the system can be given and the system is to be taken as it is, only avoiding high degrees of overall recursiveness can be recommended. Yet, as is discussed later, recursiveness can be used to reduce complexity by separation into smaller but complex systems of controlled units.

\subsubsection{Combining Complexity and Heterogeneity}

The aforementioned complexity is based on the average connectivity and needs to be considered in the light of heterogeneity:

With $\alpha=\ln (K / N+1) / \ln N=\ln (\bar{k}+1) / N$ and

$\overline{k(\gamma)}=a /(\gamma-2) \quad \forall \gamma>2$ we obtain

$\alpha^{(H)}=\ln (\gamma \bar{k} /(\gamma-2)+1) / \ln N$.

Clearly can be seen to which degree the parameter of complexity becomes distorted with rising heterogeneity and reaches large values when approaching the limit of $\gamma \rightarrow 2$.

\subsection{Reducing Complexity}

According to the meteorologist Edward Lorenz (1963), who originally introduced the understanding of chaotic behavior, exactly the term of "complexity" is defined as the property, which leads to unpredictable behavior of systems. Concluded reversely, complex systems need to be avoided in order to achieve controllable systems. Generally spoken, reducing complexity is a means to make a system more predictable as it simplifies its

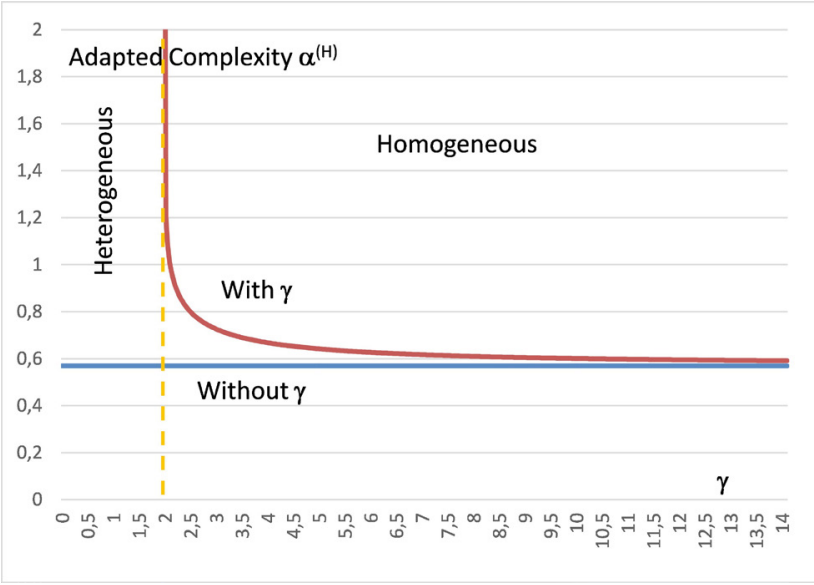

Fig. 3 Complexity $\alpha^{(H)}$ in dependence of heterogeneity $\gamma$ 
behavior (von Foerster, 1993; Malik, 2003). Using any of the given definitions of complexity, the concept of separability allows understanding this in more detail.

\subsubsection{Concept of Separability}

The tendency of breaking up a system into a set of independent superimposable units is no new understanding and has been formulated within the context of several situations (Bonacich, 1972; Fiedler, 1973). For example, the RNM-algorithm (Random Neighborhood Method) (Moody, 2001) is used to identify independent subnetworks within a network in order to treat them independently and finally superimpose their outcome. Also, the principle of division of work follows the same idea. A set of work to be done is assigned to different units as independent tasks but this is to be paid with an increase of coordination effort and expenses (Picot et al., 2008). As previously pointed out, complexity may be defined amongst other concepts by the increase of the consequences of a fault travelling through a network. Avoiding such cumulation is accomplished by shortening the length of the developing chains, i.e. separating the range where a fault may have consequences (Zimmermann and Eber, 2012).

\subsubsection{Formal Approach on Separability}

Local complexity, defined as $\alpha=\ln (\xi+1) / \ln N$ with $\xi=K / N$ according to Zimmermann and Eber (2012) is understood as the relative entropy of a node as a share of the maximum local entropy $\ln N$. Using the same understanding, the possible entropy $S$ of a total system allowing each element to equally influence any other element needs to be investigated in order to understand the effects of separability. The entropy of a total system is:

$$
S=\sum_{N} \ln (N-1)=N \ln (N-1) \text {. }
$$

If a system is separable, i.e. can be divided into two distinct subsystems, the possible interaction within the systems is reduced to a given fraction while the remaining overall interaction of the two subsystems is linear, i.e. additive. Assuming separation into subsystems of equal size each for illustration purposes, we obtain the entropy as a function of the number $z$ of subsystems. The first term refers to the entropy of the $N / z$ subsystems while the second term mirrors the entropy of the newly interacting subsystems.

$S=-(N / z) \ln (z / N)-z \ln (1 / z)$.
The minimum is given by the balance of reducing the entropy of the subsystems with size but increasing entropy with the rising number of still interacting subsystems:

$0=(\partial / \partial z) S \Rightarrow z_{\min }=\sqrt{N}$.

The degree of recursiveness is also reduced by separation into smaller subsystems since a significant number of loops is cut down to smaller loops with the subsystems or fewer loops through interdependencies between subsystems. Assumedly let the recursiveness utilize the complete volume of the system, i.e. the interactions distributed over the volume. If $z$ subsystems are separated, the number of interactions available for recursiveness decreases accordingly: $\beta^{(\text {unsep })} \sim N(N+1)$ and $\beta^{(s e p)} \sim(N / z)((N / z)+1)+2 z(z-1)$. Since $N$ and $z$ are expected to be large numbers we obtain furthermore: $\beta^{(u n s e p)} \sim N^{2}$ and $\beta^{(s e p)} \sim\left(N^{2} / z^{2}\right)+2 z^{2}$. The minimum of the ratio $\beta^{(s e p)} / \beta^{(\text {unsep })}$ yields the optimal separation with respect to recursiveness, provided beta being not zero and leads to: $z_{\min }=\sqrt{N} / \sqrt[4]{2}$.

In addition to this consideration of the overall recursiveness, the local recursiveness remains to be discussed. The difference would be in particular that in a very local environment no more recursiveness leading to chaotic behavior needs to be taken into account, but the recursive parameters can be analyzed and in most cases constructed in a positively utilizable way. The optimal substructure thus would be to localize recursiveness absolutely, i.e. restricted to a set of only two mutually interacting elements where the outcome can be safely dissipating $(\lambda<0)$ and therefore with $\beta^{(l o c a l)} \gg>0$ contribute starkly to stabilizing the whole system.

The issue of heterogeneity yields no optimum in terms of numbers since all these considerations refer to an average situation, which is not given with non-homogeneous systems. Therefore, the optimum state to be achieved would be a homogeneous network in general. Introducing subsystems not only has the effect of separating independent sections but also helps to understand the smaller subsystems as they demand to be more comprehensible allowing to treat them separately. This will only be the case if they are no more required to be understood as average behavior but as a well understood mechanism. So, the concept of heterogeneity becomes obsolete within the sections. This leaves the requirement of having to choose the separation so that the heterogeneity of the reduced system - comprising and thus interfacing the subsystems - is much lower and the overall situation becomes homogenous. 


\subsection{Examples and Case Studies}

In many situations, heuristic methods already utilize the principle of separability.

\subsubsection{Anti-Rigidity Measures: Time-floats and Fuzzy Logic}

Wherever complex systems need to be understood and solved, a large number of conditions for a limited number of variables needs to be met. The heuristic methods traditionally introduce approaches to weaken the conditions. In network plans the rule of using the maximum required time distance when optimizing project durations is set. Obviously being not optimal, this proceeding at least solves the contradiction of relationships aiming at a single node. Furthermore, deliberately time-floats (to be distinguished from time-floats resulting from the given relationships) are positioned in order to decouple sections of the network plan allowing delays not to pass transitions (Kerzner, 2003; Schelle et al., 2005). The same methods are applied on production volumes introducing safety margins and overproduction. Similarly, modelling interactions as fuzzy variables weakens the strict rules of interaction in order to allow for a solvable overall system, which may be slightly or strongly contradictory otherwise.

Case Study: If a set of 10 subsequent processes each following an Erlang $(r=16)$ duration distribution where the average duration is 5 days and the variance is $\sigma=1.25$ the coupling is strong, thus $\alpha=\ln (10 / 10+1) / \ln (10)=0.3$. Introducing float times of 1 day between the subsequent processes reduces coupling from $45.1 \%$ to $21.2 \%$ i.e. from $100 \%$ right hand overtime risk to $47 \%$ overtime risk. Therewith, the resulting complexity is reduced to $\alpha=\ln (0.47+1) / \ln (10)=0.167$ while a float time of 2 days leads to only $\alpha=\ln (0.15+1) / \ln (10)=0.06$.

\subsubsection{Network Plan}

A network plan being the set of activities to be consistently positioned on the time-axis is artificially restricted to being loop-less $(\beta=0)$ and thus restricted regarding its complexity. This is required based on the argument of mapping logical sequences to ranks where the cause always lies on a lower rank than the consequence. Then loops cannot exist and even if solved by iteration a worst-case maximum of $N$ iteration runs of $N$ steps each is required to assign each node the correct rank value. Classical algorithms such as FORD (Kerzner, 2003; Schelle et al., 2005) rely on this fact.

The average complexity approach allows estimating the average effort to $\xi=N^{\alpha}-1$ steps per $N$ worst-case runs where heterogeneity plays no significant role. Yet, if nodes to be calculated are picked randomly the effort rises nonlinearly with the center of gravity of the high degree nodes sitting more towards the start in contrast to the end of the causal chain. Taking the extended complexity $\alpha^{(H)}=\ln (\gamma \xi /(\gamma-2)+1) / \ln N$ and therefrom $N^{\alpha^{(H)}}=\gamma \xi /(\gamma-2)+1$ as the speed of propagation of changes through the network, at least the increase of effort can be estimated to $A=N \xi \Rightarrow A=\gamma N \xi(\gamma-2)$. Besides constant factors, this is $N \xi$ for large values of $\gamma$ proportional to $N$ as before, but rises to infinity with $\gamma$ approaching the value of 2 .

Yet, reflecting real situations circular references are indeed possible, e.g. representing the same factual relationship seen from two or more different perspectives redundantly. If known, they could be eliminated, but if not, they lead to an infinite number of iteration runs and therewith infinite results when calculating causal ranks. If iterating positions on the time-axis instead the results will be finite since redundant interdependencies lead to the same result and thus a stabilizing situation. Even more, slightly contradictory instructions lead to a virtually stable situation as the system may oscillate with low amplitudes around the fuzzy solution correctly indicating the slightly undefined true position on the time axis (Kerzner, 2003; Schelle et al., 2005; Schulte-Zurhausen, 2002; Zimmermann and Eber, 2010). In this case, $\beta \neq 0$ is required but at the same time the parameters $\lambda$ inevitably need to be real and negative or at least, if complex, leading to oscillation with a strongly limited amplitude. Since this is not always the case, such systems pose the challenge to be designed carefully in order to exhibit long-term stable behavior.

Case Study: A most simple strictly linear network clearly fulfills the requirement of being a loop-less network. With a given number of e.g. 50 activities each directly following the other we have: $\beta=0, \gamma=\infty$ and thus $\alpha=\alpha^{(H)}=\ln ((50-1) / 50+1) / \ln (50)=0.17$. This can only be simplified by further reducing the number of members of the given chain of activities. If the activities were arranged as a completely parallel set we obtain $\alpha=\ln ((50-2+50-2) / 50+1) / \ln (50)=0.27$. However, the strong central pooling node leads to a starkly inhomogeneous system $\gamma \approx 1$ where $\alpha^{(H)}=\infty$ becomes virtually infinite and no sensible statements can be issued.

\subsubsection{Tree-Structures}

Classical tree-structures are constructed in a similar way, introducing artificial restrictions in order to simplify the 
behavior. In particular, the requirements of being loopless and of unambiguous unidirectional paths from each node to the singular source-node are effecting limited complexity (Fiedler, 1973; Zimmermann and Eber, 2014). This induces some principle incompleteness since the characteristic variable to branch on is reduced to merely a single one, which does not correspond to reality. Yet, separability is made use of, based on the assumption that subnodes are only cooperating via the single super-node and do not have other interrelations.

The recursiveness $\beta=0$ clearly keeps the system small and predictable, unidirectional paths furthermore ensure short and clear lines of impact, be it responsibility and instructions (towards the leaves of a tree) or reports (towards the root). The fundamental complexity is given by the algorithms of finding the least spanning tree, where each node is connected by as few interactions as possible, implying $\xi=K / N$ minimal $\Rightarrow \alpha=\ln (\xi+1) / \ln N$ minimal .

Extending this, the parameter of heterogeneity allows optimizing tree-structures furthermore leading to the plain rule of employing nodes with a similar span of responsibility. For example, if exactly $\mu$ nodes are connected to each super-node and $l$ levels of hierarchy are present, the number of nodes will be in total

$$
N(i)=\mu^{i} \quad N=\sum_{i=0 . l} \mu^{i}=\left(\mu^{l-1}-1\right) /(\mu-1) \quad K=N-1 .
$$

The number of connections is $K=N-1$ since each node is connected to exactly one super-node except the top-node itself. Counting downward yields the same value due to the closed character of the graph. With $\xi=K / N=(N-1) / N$ the fundamental complexity is fairly small for larger systems $\alpha=\ln ((N-1) / N+1) / \ln N \simeq \ln 2 / \ln N$. Any deviation from a constant responsibility span $\mu$ changes not much of the structure itself but leads to rising heterogeneity, which should be avoided. This is only a very minor requirement since a tree-structure is already reduced to an optimal shape as far as possible.

Case Study: Let a tree-structure represent the responsibility for certain units, e.g. $N=50$. Since responsibility can neither be operated in loops, nor can deal with double paths, the tree is the only available structure leading to the parameters $\beta=0, \gamma=\infty, \alpha=\alpha^{(H)}=\ln 2 / \ln 50=0.17$. However, the physical decomposition of a building would follow a similar tree-structure with the same parameters, but the constructor would be forced to limit the numerous existing interactions of the elements to the few options permitted by the tree.

\subsubsection{Control-loops}

Inherent dependencies, e.g. the necessity of construction parts to fit, are traditionally not implemented in maps of the system but defined by design ("Gestaltungsplanung") (Zimmermann et al., 2014). Thus, they are expected to be fulfilled without further activity. Yet, this dependency is still given and the interaction is active and possibly turns out to be crucial if not matching perfectly. On this background, a fairly complex system is treated in a starkly simplified manner by merely ignoring the given complexity.

On the one hand, treating the complete system accordingly would present the correct parameters of complexity, heterogeneity and recursiveness. On the other hand, methods are required to construct the system in a way, which maintains the expected simplicity. This is accomplished by the introduction of control-loops. Additional elements (so-called "control processes") are introduced besides each critical element ensuring the accuracy of particular variables within the given margins. Therewith the strong dependency of the consuming node on the quality of the providing node is completely broken, the system largely decoupled into numerous fairly small independent subsystems. This is valid as long as the resources required to ensure the controlling are not coupled themselves and add another dependency. Based on the strength of the controlling units additional effects like the stabilizing behavior and the time constants to stabilize the result come into play (Zimmermann and Eber, 2012). The subsystems tend to behave like coupled oscillators, where the transfer of oscillations through the network needs to be observed very carefully. Furthermore, fast oscillations are introduced by fast regulators leading to the necessity of damping the behavior by low-pass filtering of the network, i.e. dissipation by cumulating local values and thus a lower reaction time.

If all possible interactions of a complex system were separated by introducing $N$ additional control-loops, the resulting system may be treated as a new system comprising $N$ pairs of elements being perfectly controlled and held at constantly fitting values. Thus, the local $\beta \gg 0$ are highly recursive but due to the very local character of the loops well dampened and under control. Then, all interactions of the remaining system would vanish at least to a degree of control $\eta$ ranging in [0..1], the heterogeneity would be unchanged as well as the inherent $\beta$. Only the number of (=sum of weighted) interactions would be reduced by a factor of $\eta$ while probably an additional number of $N \eta$ interactions would appear due to 
the dependency of the required resources for each control loop on the total effort.

With $K^{(C)}=K(1-\eta)+N \eta=K+(N-K) \eta$ we obtain $\alpha^{(C)}=\ln ((1+\xi)+(1-\xi) \eta) / \ln N$. In total, mainly independent of $\xi$, a control degree of about $\eta \approx 0.9$ is required to bring the complexity down to $50 \%$. In particular needs to be denoted that there is no minimum detectable indicating complete control to be the optimal improvement to a system.

Case Study: A set of 100 tightly interacting elements with $\xi=3$ leads to $\beta=0$ and $\gamma=\infty$ therewith to complexity $\alpha=\ln (300 / 100+1) / \ln (100)=0.3$. Introducing additional control elements for each value adds another 100 supervising elements and two further interactions each for control. Thus, we obtain a new value of complexity which does not change much: $\alpha=\ln ((300+2 \cdot 100) /(100+100)+1) / \ln (100+100)=0.23$. However high recursiveness is introduced since the control elements refer to the controlled elements and vice versa leading possibly to $\beta=1$ where $\alpha^{(H)}$ escalates. Yet, it is known (since the construction of control requires this to be so), that the respective exponents $\lambda$ are strictly negative, the subsystems formed by an element plus the controlling element comprise all the respective recursiveness and can be treated as completely stable subsystems, safely providing the given values. Thus, the system formed by the stable subsystems is no more dependent and we obtain vanishing complexity: $\alpha=\ln ((300 \cdot 0) /(100)+1) / \ln (100)=0$.

\section{Conclusion}

Organizational structures, e.g. for a Real Estate or Construction project, cannot be predefined in general but need to be set up according to the given situation.

On the one hand, the situation is determined e.g. by a social or technical environment, a market, a specific method or task, or a structure inherited from the past. Then, a meticulous analysis is required to understand and predict its future

\section{References}

Barabási A.-L., Albert, R. (1999) "Emergence of Scaling in Random Networks", Science, 286(5439), pp. 509-512. https://doi.org/10.1126/science.286.5439.509

Bonacich, P. (1972) "Factoring and weighting approaches to status scores and clique identification", The Journal of Mathematical Sociology, 2(1), pp. 113-120.

https://doi.org/10.1080/0022250X.1972.9989806

Caldarelli, G., Vespignani, A. (2007) "Preliminaries and Basic Definitions in Network Theory", In: Large Scale Structure and Dynamics of Complex Networks: From Information Technology to Finance and Natural Science, Complex Systems and Interdisciplinary Science Series, 1st ed., World Scientific Publishing Co. Pte. Ltd., Singapore, Singapore, pp. 5-16.

https://doi.org/10.1142/9789812771681_0002 behavior as are actions, performance and conduct. In terms of systems theory this is its general stability and sensitivity behavior based not so much on details but on central parameters like complexity, heterogeneity and recursiveness proposed here. This will principally allow judging the value or risk of any engagement to the given situation or project and enable to make proposals of improvement. At least critical hotspots of the project can be detected easily and special attention directed to these, which may turn out to be crucial for large and tightly constructed projects.

On the other hand, systems, i.e. organizations, are unique to each project and therefore to be constructed explicitly for the particular needs. Since projects are defined to be non-recurrent and non-repetitive, exactly the fitting organization is required to cover the risks of unknown variables and situations by its ability to treat them positively and therewith lead the project to success. Thus, risk management is the property of an organization to become independent of lacking specific knowledge of particular variables. Therefore, parameters like complexity, heterogeneity and recursiveness are the basis for any estimation of the sensibility of the organization towards changes of variables and determine the behavior, i.e. the stability of the crucial results. Thus, organization structures need to be constructed with a particular focus on such parameters and optimized with respect to these prior to being set in operation.

In short, we propose, based on the formal proof of the heuristically well known rules that any organization or structure must be exhibit the least possible complexity $\alpha, \alpha^{(C)}, \alpha^{(R)}, \alpha^{(H)}$, which can be achieved by constructing as many subsystems as possible, mainly independent from each other and subjected to strong local controlling mechanisms, where again resources need to be independent of each other. Only after this, classical optimization methods may be applied to the given system without the need to reconfigure fundamental pre-settings.

Fiedler, M. (1973) "Algebraic connectivity of graphs", Czechoslovak Mathematical Journal, 23(2), pp. 298-305.

Gordon, T., Helmer, O. (1964) "Report on a Long-range Forecasting Study", 1st ed., The RAND Corporation, Santa Monica, California, USA.

Haken, H. (1983) "Synergetik: Eine Einführung", (Synergetics: An Introduction) 3rd revised and enlarged ed., Springer Verlag, Berlin, Heidelberg, Germany. (in German)

Kerzner, H. (2003) "Project Management: A Systems Approach to Planning, Scheduling, and Controlling", 8th ed., Wiley, Berlin, Germany.

Lewis, J. P. (2002) "Fundamentals of Project Management", 2nd ed., American Management Association, New York, USA.

Lorenz, E. N. (1963) "Deterministic Nonperiodic Flow", Journal of the Atmospheric Sciences, 20(2), pp. 130-141.

https://doi.org/10.1175/1520-0469\%281963\%29020<0130 $\% 3 \mathrm{ADNF}>2.0 . \mathrm{CO} \% 3 \mathrm{~B} 2$ 
Luhmann, N. (1984) "Soziale Systeme: Grundriß einer allgemeinen Theorie", (Social Systems: Outline of a General Theory) 1st ed., Suhrkamp Verlag, Frankfurt am Main, Germany. (in German)

Malik, F. (2003) "Systemisches Management, Evolution, Selbstorganisation", (Systemic Management, Evolution, Selforganisation) 3rd ed., Haupt Verlag, Bern, Switzerland. (in German)

Moody, J. (2001) "Peer Influence Groups: Identifying Dense Clusters in Large Networks", Social Networks, 23(4), pp. 261-283. https://doi.org/10.1016/S0378-8733(01)00042-9

Newman, M. E. J. (2003) "The Structure and Function of Complex Networks", SIAM Review, 45(2), pp. 167-256. https://doi.org/10.1137/S003614450342480

Picot, A., Dietl, H., Franck, E. (2008) "Organisation - Eine ökonomische Perspektive", (Organisation - an Economical Perspective) 5th rev. ed., Schäffer-Poeschel, Stuttgart, Germany. (in German)

Schelle, H., Ottmann, R., Pfeiffer, A. (2005) "Project Manager", 2nd ed., GPM Deutsche Gesellschaft für Projektmanagement, Nürnberg, Germany.

Schulte-Zurhausen, M. (2002) "Organisation", (Organisation) 3rd ed., Verlag Franz Vahlen, München, Germany. (in German)

Shannon, C. E. (1948) "A Mathematical Theory of Communication", The Bell System Technical Journal, 27(3), pp. 379-423. https://doi.org/10.1002/j.1538-7305.1948.tb01338.x

Straub, D. (2014) "Value of Information Analysis with Structural Reliability Methods", Structural Safety, 49, pp. 75-86. http://doi.org/10.1016/j.strusafe.2013.08.006

Strogatz, S. H. (2001) "Exploring complex networks", Nature, 410, pp. 268-276. https://doi.org/10.1038/35065725

von Bertalanffy, L. (1969) "General Systems Theory: Foundations, Development, Applications", 2nd revised ed., George Braziller Inc., New York, USA. p. 54.

von Foerster, H. (1993) "Wissen und Gewissen", (Knowledge and Conscience) 9th ed., Suhrkamp Verlag, Frankfurt am Main, Germany, p. 73.

Wasserman, S., Faust, K. (1994) "Social Network Analysis: Methods and Applications", 1st ed., Cambridge University Press, Cambridge, United Kingdom.

White, D. R., Owen-Smith, J., Moody, J., Powell, W. W. (2004) "Networks, Fields and Organizations: Micro-Dynamics, Scale and Cohesive Embeddings", Computational and Mathematical Organization Theory, 10(1), pp. 95-117. https://doi.org/10.1023/B:CMOT.0000032581.34436.7b
Wiener, N. (1992) "Kybernetik. Regelung und Nachrichtenübertragung im Lebewesen und in der Maschine", (Cybernetics or Control and Communication in the animal and the machine) 3rd ed., Econ Verlag, Düsseldorf, Germany, (in German)

Zimmermann J., Eber, W. (2010) "Simulation - von der prozeduralen zur objektorientierten Modellierung", (Simulation - from Procedural to Object-Oriented Modelling) In: Tagungsband zum SimulationsWorkshop Bauhaus-Universität Weimar, Modellierung von Prozessen zur Fertigung von Unikaten, (Proceedings of Simulation Workshop, Bauhaus-University Weimar, Modelling Processes for the Production of Unique Specimens) Weimar, Germany. pp. 37-46. (in German)

Zimmermann, J., Eber, W. (2012) "Development of Heuristic Indicators of Stability of Complex Projects in Real Estate Management", In: The $7^{\text {th }}$ International Scientific Conference "Business and Management 2012", Vilnius, Lithuania, pp. 1269-1277. https://doi.org/10.3846/bm.2012.163

Zimmermann, J., Eber, W. (2014) "Mathematical Background of Key Performance Indicators for Organizational Structures in Construction and Real Estate Management", Procedia Engineering, 85, pp. 571-580.

https://doi.org/10.1016/j.proeng.2014.10.585

Zimmermann, J., Eber, W., Tilke, C. (2014) "Unsicherheiten bei der Realisierung von Bauprojekten - Grenzen der wahrscheinlichkeits-basierten Risikoanalyse", (Uncertainty of decisions in the construction sector - Limitations of probability based risk analysis) Bauingenieur, 89(6), pp. 272-282. (in German)

Zimmermann, J., Eber, W. (2017) "Criteria on the Value of Expert's Opinions for Analyzing Complex Structures in Construction and Real Estate Management", In: Creative Construction Conference 2017, CCC 2017, Primosten, Croatia, pp. 335-342. https://doi.org/10.1016/j.proeng.2017.07.208

Zimmermann, J., Eber, W. (2018) "Optimizing Organizational Structures in Real Estate and Construction Management", In: Creative Construction Conference 2018, CCC 2018, Ljubljana, Slovenia, pp. 602-610.

https://doi.org/10.3311/CCC2018-080 